\title{
COMMENTARY
}

\section{Syntrophy in microbial fuel cells}

\author{
Jan Dolfing
}

The ISME Journal (2014) 8, 4-5; doi:10.1038/ ismej.2013.198; published online 31 October 2013

Syntrophy has a pivotal role in the microbial degradation of organic compounds in methanogenic ecosystems (McInerney et al., 2009). Methanogenic degradation of organic compounds is a sequential process: a series of organisms is involved in the various conversion steps of these compounds into methane and carbon dioxide (Dolfing, 1988). Typically, the product of one conversion step is the substrate for the next organism in the chain; each organism lives off the waste product(s) of its predecessor. Their defining characteristic as it relates to syntrophy is that in many of these associations the producer is critically dependent on the activities of the consumer (Schink and Stams, 2006): degradation of short chain volatile fatty acids like propionate and butyrate is only sustainable if the electrons produced in the process are removed by other organisms (Dolfing, 2013). This concept was first put forward by Bryant et al. (1967) who famously invoked thermodynamics to rationalize their observation that ethanol degradation could only sustain growth of an ethanol degrader if the hydrogen produced in the process is removed by a hydrogenotrophic methanogen. Since then we have learned that interspecies hydrogen transfer is not the only mechanism to facilitate syntrophy: interspecies formate transfer and direct interspecies electron transfer (DIET) are distinct alternatives to the classical pathway (Stams and Plugge, 2009; Summers et al., 2010). Obviously, the very core value of syntrophy-the critical interdependency between producer and consumer-has not been challenged by this changing perspective. That is, until recently. Earlier this year Kimura and Okabe (2013) reported that Geobacter sulfurreducens PCA can oxidize acetate in what the authors labelled 'a syntrophic cooperation' with Hydrogenophaga sp. strain AR20 in conjunction with an electrode as the final electron acceptor. However, close reading of their paper reveals that $G$. sulfurreducens PCA does not require the presence and activity of strain AR20 to perform this feat: in pure culture G. sulfurreducens PCA can also oxidize acetate in conjunction with an electrode as the final electron acceptor; the organism can grow on this reaction, as expected (Bond and Lovley, 2003). Thus G. sulfurreducens PCA does not critically depend on its partner, and the association between the two organisms is therefore not syntrophic. For the co-culture to be syntrophic, it would require that G. sulfurreducens ceases to be an electrogen, or more precisely ceases to be able to use electrodes as electron acceptor. The data put forward by Kimura and Okabe (2013) provide no evidence that this indeed the case, though they do indicate that G. sulfurreducens PCA benefits from the presence of Hydrogenophaga sp. strain AR20. It will be interesting to see to what extent the anode potential modulates the interactions between these two electrogens, and it is tempting to speculate that applying the 'optimal' electrode potential may coax the co-culture into syntrophy.

Morris et al. (2013) recently noted that in the literature on anaerobic ecosystems syntrophy is traditionally described in detailed mechanistic terms, making explanations of the concept rather wordy. They therefore propose to define syntrophy as 'obligately mutualistic metabolism'. This definition indeed covers the very core value of syntrophy highlighted above-the critical interdependency between producer and consumer-but will not alleviate the need to highlight the (thermodynamic) rational behind this interdependency in anaerobic ecosystems. Interestingly, the host-bacterial mutualism in the human intestine (Bäckhed et al., 2005) and area of much current research (for a recent review, see for example Sommer and Bäckhed, 2013) also seems to fall within the realm of syntrophy.

It is to be expected that the recent eye-openers on DIET and DIET-based syntrophic growth (Summers et al., 2010; Shrestha et al., 2013) will not only lead to more exciting work on this mechanism but will also give new impetus to the traditionally more biochemical and thermodynamical-oriented research on syntrophy via interspecies hydrogen and formate transfer (Stams and Plugge, 2009; Sieber et al., 2012). Surprisingly, little is known about the kinetics behind syntrophic interactions and the interplay between kinetics and thermodynamics (Dolfing and Tiedje, 1986; Dwyer et al., 1988; Stams et al., 2006). Microbial fuel cells are promising tools to tackle those issues, but traditional chemostat studies offer perspective as well, not only for studies on syntrophy but also for studies on other types of interactions like those between acetogenesis and methanogenesis (Lever, 2012; Oren, 2012). Given the long running times needed to obtain comprehensive data sets with chemostat 
systems, it seems prudent to start such studies with some modelling work to delineate at which dilution rates insightful results can be expected (Xu et al., 2011; Dolfing and $\mathrm{Xu}, 2012$ ).

\section{Acknowledgements}

This work was funded by the Engineering and Physical Sciences Research Council UK (Grant no. EP/E057462/1) and the Biotechnology and Biological Sciences Research Council UK (Grant no. BB/K003240/1). I gratefully acknowledge Ian Head for constructive comments.

$J$ Dolfing is at School of Civil Engineering and Geosciences, Newcastle University, Newcastle, UK E-mail: jan.dolfing@ncl.ac.uk

\section{References}

Bäckhed F, Ley RE, Sonnenburg JL, Peterson DA, Gordon JI. (2005). Host-bacterial mutualism in the human intestine. Science 307: 1915-1920.

Bond DR, Lovley DR. (2003). Electricity production by Geobacter sulfurreducens attached to electrodes. Appl Environ Microbiol 69: 1548-1555.

Bryant MP, Wolin EA, Wolin MJ, Wolfe RS. (1967). Methanobacillus omelianskii, a symbiotic association of two species of bacteria. Arch Mikrob 59: 20-31.

Dolfing J. (1988). Acetogenesis. In: Zehnder AJB (ed.) Biology of Anaerobic Microorganisms. John Wiley and Sons Inc: New York, pp 417-468.

Dolfing J. (2013). Syntrophic degradation of propionate via butyrate: A novel window of opportunity under methanogenic conditions. Appl Environ Microbiol 79: $4515-4516$.

Dolfing J, Tiedje JM. (1986). Hydrogen cycling in a threetiered microbial food-web growing on the methanogenic conversion of 3-chlorobenzoate. FEMS Microbiol Ecol 38: 293-298.

Dolfing J, Xu A. (2012). Methanogens are the weakest link: energetics and kinetics of syntrophic associations with and without methanogens. In: Abstracts ISME14 14th International Symposium on Microbial Ecology. 19-24 August 2012. Session IS36 on Syntrophy and electron flow in microbial communities: Copenhagen, Denmark.

Dwyer D, Weeg-Aerssens E, Shelton DR, Tiedje JM. (1988). Bioenergetic conditions of butyrate metabolism by a syntrophic anaerobic bacterium in coculture with hydrogen-oxidizing methanogenic and sulfidogenic bacteria. Appl Environ Microbiol 54: 1354-1359.

Kimura ZI, Okabe S. (2013). Acetate oxidation by syntrophic association between Geobacter sulfurreducens and a hydrogen-utilizing exoelectrogen. ISME $J$ 7: 1472-1482.

Lever MA. (2012). Acetogenesis in the energy-starved deep biosphere - a paradox? Front Microbiol 2: 284.

McInerney MJ, Sieber JR, Gunsalus RP. (2009). Syntrophy in anaerobic global carbon cycles. Curr Opin Biotechnol 20: 623-632.

Morris BEL, Henneberger R, Huber H, Moissl-Eichinger C. (2013). Microbial syntrophy: interaction for the common good. FEMS Microbiol Rev 37: 384-406.

Oren A. (2012). There must be an acetogen somewhere. Front Microbiol 3: 22.

Schink B, Stams AJM. (2006). Syntrophism among prokaryotes. Prokaryotes 2: 309-335.

Shrestha PM, Rotaru A-E, Aklujkar M, Liu F, Shrestha M, Summers ZM et al. (2013). Syntrophic growth with direct interspecies electron transfer as the primary mechanism for energy exchange. Environ Microbiol Rep 5: 904-910.

Sieber JR, McInerney MJ, Gunsalus RP. (2012). Genomic insights into syntrophy: The paradigm for anaerobic metabolic cooperation. Annu Rev Microbiol 66: $429-452$.

Sommer F, Bäckhed F. (2013). The gut microbiota masters of host development and physiology. Nat Rev Microbiol 11: 227-238.

Stams AJM, de Bok FAM, Plugge CM, van Eekert MHA, Dolfing J, Schraa G. (2006). Exocellular electron transfer in anaerobic microbial communities. Environ Microbiol 8: 371-382.

Stams AJM, Plugge CM. (2009). Electron transfer in syntrophic communities of anaerobic bacteria and archaea. Nat Rev Microbiol 7: 568-577.

Summers ZM, Fogarty HE, Leang C, Franks AE, Malvankar NS, Lovley DR. (2010). Direct exchange of electrons within aggregates of an evolved syntrophic coculture of anaerobic bacteria. Science 330: 1413-1415.

Xu A, Dolfing J, Curtis TP, Montague G, Martin E. (2011). Maintenance affects the stability of a two-tiered microbial ‘food-chain'? J Theor Biol 276: 35-41. 